\title{
Dinámica de la cadena global aeroespacial: un análisis de teoría de grafos
}

\author{
Dynamics of the global aerospace chain: \\ An analysis of graph theory

\section{Héctor Eduardo Díaz Rodríguez*, Mario Alberto Morales Sánchez, Seyka Sandoval Cabrera}

\author{
Universidad Nacional Autónoma de México, México \\ Recibido el 23 de enero de 2019; aceptado el 29 de agosto de 2019 \\ Disponible en Internet el: 4 de septiembre de 2019
}

\section{Resumen}

Este estudio analiza mediante teoría de grafos y con la metodología de cadenas globales de valor, los cambios ocurridos en el comercio aeroespacial global en el último cuarto de siglo. Se identifican patrones de comercio en los que los países líderes, si bien descentralizan una parte de la producción, dichos cambios no alteran sus condiciones de liderazgo. Países como Canadá y China, que no se han integrado a la cadena global de valor de la industria en forma tradicional, se han convertido líderes, mientras que países como México que se han integrado en la cadena siguiendo la teoría del upgrading tecnológico, se han quedado rezagados a la producción de los segmentos más bajos de valor de la cadena.

Código JEL: F14, O25, L93

Palabras clave: Industria aeroespacial; Cadenas globales valor; Teoría de grafos

\footnotetext{
*Autor para correspondencia

Correo electrónico: diazrhe@economia.unam.mx (H. E. Díaz Rodríguez).

La revisión por pares es responsabilidad de la Universidad Nacional Autónoma de México.

http://dx.doi.org/10.22201/fca.24488410e.2020.2418

0186- 1042/@ 2019 Universidad Nacional Autónoma de México, Facultad de Contaduría y Administración. Este es un artículo Open Access bajo la licencia CC BY-NC-SA (https://creativecommons.org/licenses/by-nc-sa/4.0/)
} 


\begin{abstract}
This study aims to analyze, the changes that have taken place in global aerospace trade in the last 25 years, using a global chains value perspective and theory of graphs. Trade patterns are identified in which the leading countries, although they decentralize the production, those changes do not alter their leadership conditions. Countries such as Canada and China, which have not integrated into the global chain of the industry in a traditional way, have become in leaders, while countries such as Mexico that have been integrated in the chain following the theory of technological upgrading, have remained lagged behind the production of the lower value segments.
\end{abstract}

JEL code: $\mathrm{F} 14, \mathrm{O} 25, \mathrm{~L} 93$

Keywords: Aerospace industry; Global value chains; Graph theory

\title{
Introducción
}

La industria aeroespacial (IA) es una de las más dinámicas a nivel global; Airbus (2018) estima que el número de aviones requeridos para el traslado de personas y mercancías en 20 años, será de poco menos del doble de la flota total existente en 2018. Ello generará no solo cambios cuantitativos en la producción, sino también en la configuración de la producción de bienes y servicios vinculados con la industria. En los últimos años, la mayor demanda de vuelos, el incremento del tráfico aéreo, cambios en la composición de las cadenas globales de producción del sector, y las políticas nacionales de impulso al ramo, han modificado de manera sustancial la producción y el comercio aeroespacial.

El presente estudio tiene el objetivo de analizar los cambios ocurridos en la IA mundial durante los últimos 24 años, así como las políticas nacionales que los generaron. Las fases del proceso de producción de una aeronave van desde la investigación, el desarrollo, diseño, producción, ensamblaje y continúan con la comercialización y servicios y posventa. Debido a que cada una de esas etapas no ocurre en una sola ubicación geográfica, se define a la IA como una cadena global de producción; por ello, resulta conveniente que la base teórica para realizar el análisis del sector sea la de Cadenas Globales de Valor (CGV).

La metodología que se utiliza para analizar de forma sintética los flujos comerciales de la IA en el mundo, es la teoría de grafos o redes; ella en principio, permite analizar los principales nodos comerciales, la dirección de los flujos, el saldo comercial de cada país, los socios comerciales y los cambios en los patrones de comercio a lo largo del tiempo. Esta metodología aporta insumos analíticos para entender las estrategias productivas y comerciales seguidas por los líderes. El trabajo se estructura de la siguiente manera: en la sección siguiente, se 
desarrolla el planteamiento teórico de las CGV y se hace una revisión de los estudios que, desde esta perspectiva, han analizado a la IA; en la sección tercera se desarrolla la metodología de grafos o redes. En la cuarta sección se analiza, aplicando la metodología, el comercio aeroespacial mundial en tres momentos; 1995, 2005 y 2017. La selección de años permite dar seguimiento a los cambios más significativos en los patrones de comercio aeroespacial en el mundo, así como entender las políticas nacionales que los generaron. En esta sección se hace especial seguimiento a los países líderes y a México. Por último, en la sección 4 se ofrecen las conclusiones derivadas del estudio.

\section{Revisión de la literatura}

La liberalización económica mundial que comenzó a mediados de los años setenta en todo el mundo, dio origen a cambios importantes que modificaron de manera sustancial las relaciones de comercio entre países, generalmente asociadas a ventajas competitivas y patrones de desarrollo histórico, y las sustituyó por relaciones de intercambio de bienes intermedios, maquinaria y tecnología entre empresa de alcance transnacional. Ello dio origen a un intercambio global organizado en cadenas de valor, que actualmente representa poco menos del 80 por ciento del comercio a nivel mundial. La tendencia general de este patrón comercial es la incorporación de una alta cantidad de importaciones por unidad exportada de producto. (Dickens 2011, pp. 7; Gereffy, et al., 2005; 2016; Ortiz-Ospina et al, 2018).

Los cambios en los patrones comerciales, modificaron también la idea de desarrollo por parte de las economías emergentes, que sustituyeron los antiguos patrones de sustitución de importaciones, por el desarrollo de ciertas industrias ligadas a la dinámica internacional de las CGV, con la expectativa de que dicha integración produciría derramas positivas sobre el crecimiento, principalmente liderado por el sector exportador. En este contexto, se parte de la idea de las transacciones intraempresa son más baratas y eficientes (al no someterse a los costos de las políticas comerciales) lo que reduce los costos de transacción haciendo más eficientes los procesos productivos (Williamson, 1979; 1975); lo cual implica cambios en la estructura de gobierno de las empresas, es decir cambios en la forma funcional interna para la producción de bienes y servicios (Gereffi, et al., 2005; Williamson, 1979).

El concepto de cadena de valor se centra en analizar el conjunto de actividades, procesos y fases que realizan las empresas para la producción de bienes y servicios. Estos procesos van desde investigación y desarrollo, diseño, producción, comercialización, venta, post venta, reciclaje, etc. El concepto busca analizar la interacción entre compradores y productores que 
inciden directamente en la gobernanza de la cadena y dan lugar a una estructura industrial determinada (Gereffi et al., 1994; 1999; 2005).

El objetivo de este enfoque es analizar cuestiones de reorganización industrial, coordinación, gobernanza y poder en la cadena que dan lugar a determinada distribución de la producción del valor a escala global (Gereffi et al, 2005), al tiempo de permitir el desarrollo de las herramientas analíticas que permitan, "generar instrumentos de política eficaces, relacionados con el ascenso industrial, el desarrollo económico, la creación de empleos y la reducción de la pobreza" (Gereffy, et al., 2005, pp. 79; 2018).

La metodología permite establecer una orientación geográfica en una estructura insumo-producto e identificar a los actores centrales y empresas líderes, así como su estructura e interacción con el contexto productivo y de políticas públicas. Analiza además el contexto institucional local de los agentes integrados en la cadena, así como los factores determinantes para establecer una estrategia de escalamiento productivo (Gereffi et al, 2016). El análisis de las instituciones y organizaciones involucrados, permite visualizar las restricciones que las empresas locales enfrentan en la industria global, lo cual abre la posibilidad de poner en marcha modificaciones en la estructura productiva interna y en el diseño institucional que permitan fortalecer a los proveedores locales incorporados en la cadena de valor. Las restricciones identificadas en diversos estudios constituyen rezagos industriales históricos o de política pública en la estructura productiva de los países, en su infraestructura, entorno empresarial, y en las instituciones que sustentan dicha estructura. Al identificar estas restricciones, se facilita su atención en un marco de diseño de determinadas políticas de desarrollo productivo (PDP) que permitan integrar a las organizaciones locales con las cadenas de valor para establecer posibilidades de escalamiento (Gereffi, 2018).

Una forma de evaluar a la industria aeroespacial mediante el enfoque de CGV, es utilizando el modelo de Alianzas Transnacionales (ACT) como política pública de desarrollo, y analizar si la configuración de una estrategia de escalamiento tiene implicaciones positivas sobre la posición que ocupan los países dentro de la cadena o en el comercio internacional. En este contexto, es importante analizar si el liderazgo de la cadena y por lo tanto, las posibilidades de los países en desarrollo de escalar en la misma, son factibles para modificar la estructura de gobernanza, o si por el contrario, los segmentos más rentables de la cadena presentan estructuras de organización concentradas en pocas empresas ubicadas en los países líderes que protegen con barreras a la entrada la propiedad del conocimiento, o bien, mediante acuerdos comerciales que prohíben la competencia directa. Lo anterior, implica que la estrategia de escalamiento se encuentra acotada desde el origen, y que los países que la siguen no podrán ubicarse en la producción de los segmentos de mayor valor agregado. Si ello ocurre, el modelo 
de alianzas transnacionales prolongará la brecha en la cadena, profundizando las asimetrías mediante requerimientos específicos en un marco de una alta regulación en materia de derechos de propiedad, certificaciones y estandarización de procesos; a nivel de país, pueden producirse cambios relativos en el liderazgo de los principales actores, pero no permite la entrada de nuevos competidores.

En el caso particular de la industria aeroespacial, el conocimiento se encuentra restringido por un conjunto de regulaciones y certificaciones que constituyen la principal barrera a la entrada. De acuerdo con Deloitte (2018), las empresas líderes de la IA de acuerdo con sus ingresos de 2017 fueron Boeing, Airbus Group, Lockheed Martin, General Dynamics, United Technologies, GE Aviation, Northrop Grumman, BAE Systems, Raytheon y Safran. Este conjunto de empresas concentra más del $60 \%$ de los ingresos a nivel mundial, y son responsables del diseño, ensamble final y la entrega de aeronaves (Sturgeon, et al., 2013).

A nivel nacional, en 1995 el 80\% del comercio aeroespacial global se concentraba en 5 países (Estados Unidos, Alemania, Japón, Francia y Reino Unido); en 4 países para 2005 (Estados Unidos, Alemania, Canadá y Reino Unido), y en 6 países para 2017 por la entrada de China y de Canadá en la producción de diversas aeronaves y componentes con una estrategia diametralmente distinta a la propuesta por la metodología de CGV, lo que representa una muestra significativa de la escasa movilidad a nivel país que permite dicha estrategia.

El liderazgo se vincula directamente a los derechos de propiedad sobre el diseño, el cual se basa en estrategias de investigación y desarrollo (I+D) y termina, en última instancia, definiendo cada una de las etapas de la cadena mediante especificaciones técnicas de los sistemas que integran la aeronave. Por ello, los segmentos de la cadena en los que los países en desarrollo tienen cabida, se encuentran sujetos a las especificaciones técnicas de los primeros, imposibilitando así el escalamiento real.

Lo mismo sucede con la educación y la capacitación para la integración en la cadena; esta se limita a atender las necesidades de las empresas líderes en el contexto de requerimientos técnicos y certificaciones muy específicas. En el caso de México, la influencia de las empresas trasnacionales (ET) es significativa por lo que se ha desarrollado un ecosistema productivo en torno a las necesidades de las empresas líderes; siendo la consecuencia inmediata que las firmas no integradas quedan marginadas de la proveeduría y participación en la cadena. (Sturgeon, et al, 2011; Sandoval, 2013).

La estrategia seguida por México parte de un planteamiento analítico que supone que entre mayor sea el volumen de exportaciones, mayor será el crecimiento económico; si bien en términos contables esto pudiera ser acertado, en términos reales, para que ello ocurra la totalidad de la producción para exportar tendría que generarse internamente, hecho que bajo 
mercados organizados en CGV, no sucede (Sturgeon, 2011). Si bien, ello se reconoce, la estrategia de integración busca desarrollar capacidades en las empresas mexicanas que les permitan escalar en red hacia segmentos de mayor valor dentro de la cadena (Sturgeon y Memedovic, 2011).

A nivel nacional, existe un conjunto amplio de estudios analíticos de la industria aeroespacial; sin embargo, estos tienden a centrarse en aspectos de dimensión espacial (estudios regionales o estatales), o bien, en las características y competencias de la mano de obra del sector; tal es el caso de los estudios de Hualde et al (2007) y Salinas-García (2012). Algunos otros analizan las capacidades de innovación, competitividad y transferencia de conocimiento (Brown et al, 2013; Flores et al, 2017; Cypher et al, 2013) y otro grupo de estudios tiende a centrarse en aspectos históricos y de desarrollo del sector aeroespacial (Casalet, 2013; López Galindo et al, 2018). Del conjunto de estos trabajos, se desprenden algunos de los principales problemas que, a nivel interno, enfrenta la IA mexicana; dentro de ellos destacan la formación de capital humano; acceso al crédito; mejoras en los flujos comerciales y vinculación e implementación de procesos de aprendizaje (Domínguez, et al., 2018; Vázquez y Bocanegra, 2018).

\section{Metodología}

Para analizar la estructura y evolución reciente del comercio aeroespacial, así como sus principales actores a nivel de país, el presente estudio utiliza la base de datos comercio mundial de la United Nations Conferece on Trade and Development Stat. Los datos son analizados mediante teoría de grafos, que permite identificar de manera sintética los principales patrones de comercio y generación de valor a nivel de mundial. Además, proporciona los insumos necesarios para que, en función de lo anterior, se puedan diferenciar las políticas industriales y comerciales de los países que dieron origen a ese patrón específico.

La teoría de grafos complementa en este estudio a la de Cadenas Globales de Valor en los siguientes aspectos; primero, permite obtener una perspectiva sintética en el mediano plazo, del cambio en el liderazgo comercial de los países que cuentan con un sector aeroespacial relativamente importante. No solo permite observar el tamaño del sector en términos del volumen comercial de cada país, sino también la situación de la balanza comercial (si esta es deficitaria o superavitaria) y en ese sentido, la estrategia que, en términos de la cadena global, sigue cada país. Además, muestra la dirección de los flujos de comercio nacionales incorporados como nodos. La ventaja de realizar el ejercicio para varios años, es que permite analizar el cambio observado en la posición de cada país, como resultado de la estrategia seguida, ya sea alineándose a la estrategia de upgrading en la CGV, o bien, como en el caso de 
Canadá y China, con estrategias diametralmente distintas. En la presente sección se describe la metodología de grafos o redes.

En las últimas décadas la aplicación de la teoría de redes en las ciencias sociales ha cobrado gran importancia debido a que a partir de dicha teoría se pueden analizar relaciones e interdependencias existentes entre conjuntos de datos, por ejemplo, países, individuos, empresas, sectores económicos, campos semánticos, etc. El análisis de redes en la ciencia económica ha sido poco explorado; sin embargo, en los últimos años ha tenido un importante auge debido a que, este tipo de análisis cualitativo permite visualizar de manera sencilla, estructuras complejas, como, por ejemplo, el diseño institucional y funcional de un sector o de una economía. En el análisis de una red se considera la estructura de las relaciones en las que cada actor (nodo) se encuentra involucrado; estos actores o nodos se describen a través de sus conexiones, las cuales sí son relevantes para la estructura de los nodos, se visualizan en función de dicha relevancia (Salomé, 2003).

Así, el análisis en red o grafos, se centra en las relaciones entre entidades y no en las entidades por sí mismas. De forma sintética, una red es un conjunto de puntos que están unidos mediante enlaces (líneas o aristas) o partir de una regla de asociación la cual, indica cómo se relacionan los nodos (Mitchell, 2009).

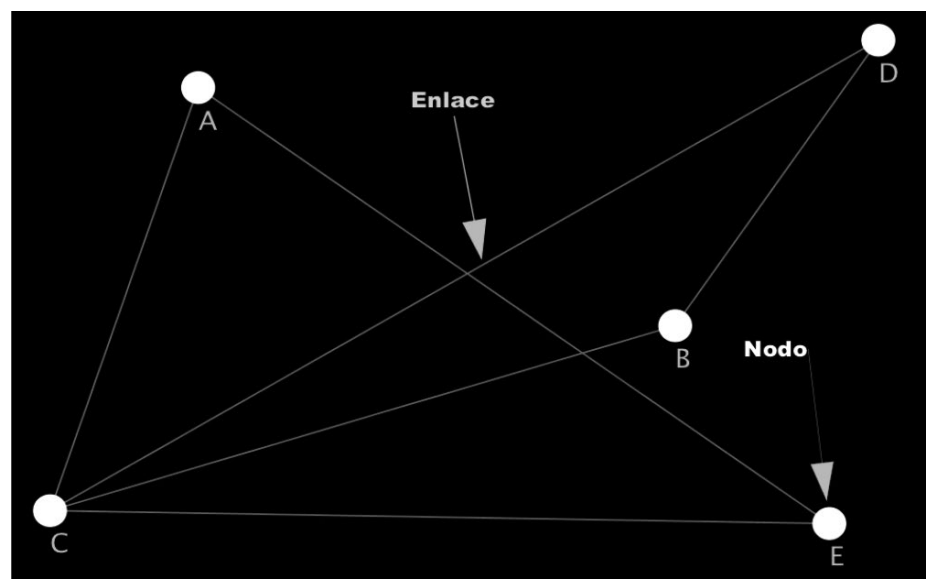

Figura 1. Diagrama general de red

Fuente: Elaboración propia con base en Mitchell, 2009.

Una red se puede denotar de manera estándar de la forma:

$$
G=(N, E)
$$


Donde $N$ es el conjunto de nodos y $E$ es el conjunto de enlaces. Por lo tanto, la figura 1 está compuesta por 5 nodos y 6 enlaces que, que pueden ser expresados como:

$$
\begin{gathered}
\{A, B, C, D, E\} \\
E=\{(A, C),(B, C),(C, D),(C, E),(D, B),(E, A)\}
\end{gathered}
$$

Una manera alternativa de representar la relación descrita en la red de la figura 1, es con una matriz $W$ conocida como matriz de adyacencia o binaria. En esta matriz $W$, se representan los nodos en las filas y columnas. Si el elemento está enlazado al elemento entonces $j$ los elementos $w_{\mathrm{ij}}$ y $w_{\mathrm{ji}}$ tendrán el valor de 1. En caso contrario, si el vínculo es inexistente, el componente de la matriz tendrá un valor de cero; lo anterior, tiene la implicación inmediata de que la matriz de adyacencia es simétrica; tiene el mismo número de filas que de columnas.

$$
\begin{aligned}
& W_{i j}=\left\{\begin{array}{l}
1, \text { si } i, j \text { están en lazados } \\
0, \text { si } i, j \text { no están enlazados }
\end{array}\right\} \\
& i, j=1, \ldots, n \\
& W=\left[\begin{array}{lllll}
0 & 0 & 1 & 0 & 1 \\
0 & 0 & 1 & 1 & 0 \\
1 & 1 & 0 & 1 & 1 \\
0 & 1 & 1 & 0 & 0 \\
1 & 0 & 1 & 0 & 0
\end{array}\right] \\
& W_{i j}=W_{j i}
\end{aligned}
$$

Un tipo distinto de análisis de redes puede establecerse mediante las redes ponderadas o grafos ponderados. Este tipo de análisis muestra los enlaces de manera valuada o ponderada (Newman, 2010). Dichos enlaces se encuentran asociados a una intensidad, representada por un valor numérico; entre mayor es la intensidad de la asociación, mayor será también la representación, medida en anchura del vínculo entre los nodos. Una representación de lo anterior, se muestra en la figura 2. 


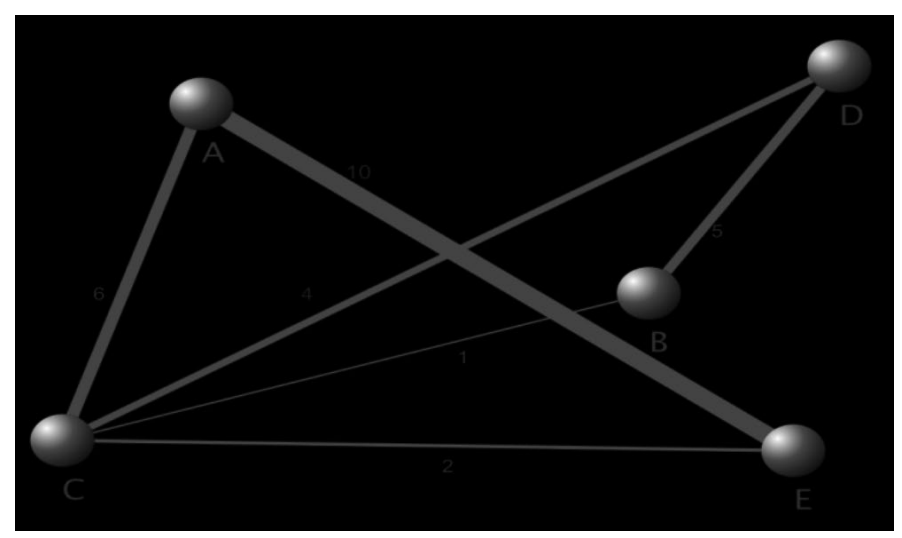

Figura 2. Representación de nodos y enlaces

Fuente: Elaboración propia con base en Mitchell, 2009.

El grosor de los enlaces de la figura 2 muestra la intensidad de la relación que existe entre dos nodos. La representación matricial de la red anterior es:

$$
Z=\left[\begin{array}{ccccc}
0 & 0 & 6 & 0 & 10 \\
0 & 0 & 1 & 5 & 0 \\
6 & 1 & 0 & 4 & 2 \\
0 & 5 & 4 & 0 & 0 \\
10 & 0 & 2 & 0 & 0
\end{array}\right]
$$

Para conocer la importancia que el nodo tiene en relación a otros, se calcula el volumen de asociación a partir del número de enlaces; a este cálculo se le conoce como centralidad de grado. Esta medida es la más simple, y mide el número de conexiones que un nodo tiene con otros nodos. Al nodo que tiene la mayor cantidad de vecinos se le conoce como "hub". La centralidad de grado es un indicador local y estático, que solo considera a los vecinos directos de un nodo.

De esta forma, para establecer la medida de centralidad de grado, se calcula la matriz de adyacencia mostrada en la ecuación 8 (Wasserman \& Faust, 1994).

$$
D_{c}=\sum_{j=1}^{n} x_{i j}=\sum_{i=1}^{n} x_{j i}
$$

\section{La industria aeroespacial en el mundo}

De 1995 a 2017, el comercio mundial de bienes y servicios de la Industria Aeroespacial (IA) ha crecido a tasas promedio de 6.7\%. Airbus (2017) estima que el en los próximos 20 años debido a la demanda de vuelos y crecimiento del comercio, el número de aviones existentes 
será del doble del actual. Pero no solo eso, sino que esa producción tiende a tener mayor tecnología que representa un mayor valor agregado, como, por ejemplo, aquella vinculada a las tecnologías de la información y comunicación (TIC), localización, seguridad y servicios de entretenimiento.

De la manera en la que los países y las firmas se posicionen para atender ese crecimiento, dependerá la configuración del escenario futuro de la producción global de la industria aeroespacial y la distribución de los beneficios derivados.

Actualmente, 5 países concentran el 82\% de la producción aeroespacial y el 85\% de los flujos comerciales en el mundo (EEUU, Alemania, China, Francia y Reino Unido). Si se considera el origen de las empresas, que por lo general desarrollan los procesos productivos fuera de su territorio, localizándose en los países o regiones que ofrezcan ventajas en términos de costos, la concentración llega a ser incluso más dramática.

La producción de las empresas más grandes de la industria aeroespacial no ocurre en uno solo lugar, sino que se integra a partir de la producción de partes de origen distinto. Las fases del proceso de producción, distribución y encadenamientos hacia adelante, como investigación y desarrollo, diseño, producción, ensamblaje, comercialización, etc., son tan variadas como los lugares en los que ocurre cada una de ellas. Por ello, su producción se define en forma de cadenas globales.

La cadena global de valor aeroespacial, se encuentra conformada por grandes fabricantes, que, por sus siglas en inglés, son denominados OEM (Original Equipment Manufacturer), los cuales pueden o no fabricar bienes intermedios, pero venden siempre productos finales, por ejemplo, motores, aviones, satélites y helicópteros (Secretaría de Economía, 2017). Un segundo elemento de la cadena de producción es el segmento de fabricantes de bienes y componentes que van directamente a la línea de ensamble final como: alas, fuselajes, trenes de aterrizaje, arneses, asientos, etc.; a este, se le conoce como Tier 1. El siguiente nivel es denominado Tier 2, que son las empresas proveedoras de partes que se integrarán a un componente del Tier1, como conectores, aislantes, cables, materiales compuestos, laminados, interruptores, etc.

Dada la concentración de la producción, el comercio y el origen de las empresas líderes del sector, las oportunidades para los países emergentes parecieran ser pocas y en el mejor de los casos, consisten en una integración a la cadena de producción como empresas tipo Tier 1 o Tier 2, segmentos de mucha menor generación de valor agregado.

Como se ha dicho anteriormente, la educación y la capacitación vinculada a la IA se desarrollan para cubrir las necesidades de integración en la cadena y se limita a atender las necesidades de las empresas líderes en el contexto de requerimientos técnicos y certificaciones 
específicas. Los beneficios a lo largo de la cadena de producción aeroespacial se distribuyen de la siguiente manera:

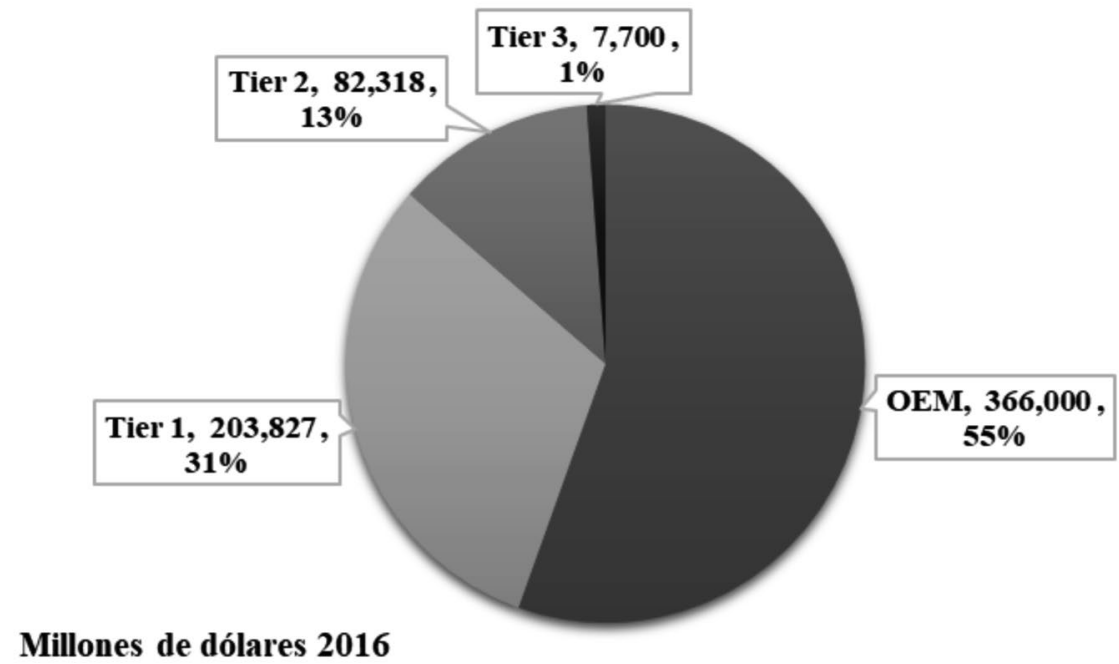

Figura 3. Distribución de los beneficios en la cadena de la IA, 2016.

Fuente: Elaboración propia con datos de Deloitte (2018).

Como se puede apreciar, el segmento de OEM es el que concentra los mayores beneficios de la cadena; si se considera que las empresas del Tier 1, generalmente también se encuentran en los países desarrollados, se puede afirmar que aquellos absorben más del 74\% de los beneficios de la cadena global de producción. En esa dirección, Estados Unidos representa en términos de regiones, si se compara con Europa, un mejor desempeño en los indicadores claves (ingresos, márgenes de operación, retorno de la inversión, etc.) (Deloitte, 2018). Como se argumentará más adelante, las perspectivas de crecimiento de la industria son positivas, tanto porque se requiere sustituir una parte importante de la flota actual, como porque es necesario satisfacer el crecimiento de la demanda mundial. Ante un escenario de crecimiento de la industria, la distribución de los beneficios asociados con crecimiento dependerá de la interacción compleja de factores internos y externos, dentro de los cuales destaca el desarrollo de capacidades por parte de las empresas domésticas, apoyados por la política industrial, no 
para integrarse en el largo plazo en la cadena, sino para crear empresas que compitan en cada segmento. En esa dirección, China ha sabido combinar política industrial, con el desarrollo de capacidades endógenas y financiamiento para tomar la delantera (Deloitte, 2018). En este terreno, México no ha desarrollado las capacidades endógenas que le permitan incursionar como nuevo productor en la cadena aeroespacial (Brown et al, 2013), ni tampoco cuenta con una política industrial en la materia que apunte en esa dirección; peor aún, su estrategia se ha circunscrito a la proveeduría de componentes en un ánimo de satisfacer las necesidades del líder de la cadena de valor. (Flores et al, 2017). Ello contrasta con lo ocurrido en países como Brasil, que construyeron industrias y empresas nacionales financiadas a partir del gasto público.

Las fusiones y adquisiciones dentro de la IA si bien son relevantes, no son la estrategia dominante; en los próximos años, la estrategia de expansión de las principales empresas será en 70\% mediante inversiones orgánicas en cooperaciones estratégicas (KPMG International, 2016).

Habiendo delineado las tendencias generales de la IA a nivel global, en el presente apartado, se describen los patrones de producción y comercio aeroespacial a lo largo de los últimos 24 años, haciendo especial énfasis en las políticas industriales y comerciales, que dieron origen a modificaciones de esos patrones en el periodo de estudio. Ello, en principio, posibilita no solo el dimensionamiento del sector en el mundo, sino también el análisis de la ubicación de México en ese contexto.

\section{Prospectiva del comercio aeroespacial en el mundo.}

Uno de los factores más relevantes para explicar el crecimiento de la demanda de vuelos a nivel mundial es el ingreso per capita. Generalmente, en la medida en la que aumenta el ingreso personal, el consumo de bienes y servicios aumenta también, por lo que se verifica un desplazamiento de la curva de demanda de la industria aeroespacial derivado principalmente del aumento en el traslado de mercancías y de personas, por lo que se observa una correlación positiva entre el incremento en el ingreso per cápita y el número de vuelos por habitante. La figura 4 muestra esta correlación en 120 países en 2017. Los datos indican que cuando el ingreso per cápita de un país es $10 \%$ mayor en relación con otro, el número de vuelos por habitante es $0.4 \%$ mayor. 


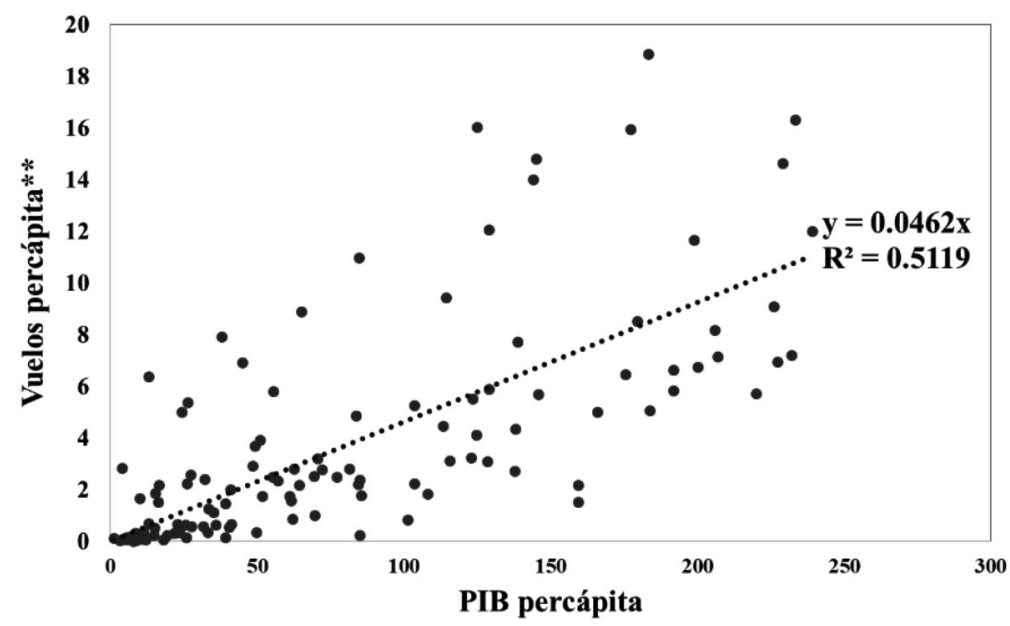

**Pasajeros originarios del país respectivo

Figura 4. Correlación entre Ingreso y vuelos

Fuente: Elaboración propia con datos de Global Market Forecast, 2018, AirBUS

El ingreso per cápita no es el único factor que explica el crecimiento del número de vuelos, sino también el incremento del comercio intra e inter regional, particularmente de los países emergentes. En esa dirección, de acuerdo con Airbus (2018), la importancia del comercio de 54 economías emergentes, que juntas, representarán el $60 \%$ del crecimiento económico mundial en los próximos 20 años, es un factor fundamental del crecimiento del tráfico aéreo futuro.

El crecimiento de la clase media, siguiente a AirBus (2018), es otro de los factores relevantes para explicar el incremento esperado del tráfico aéreo y con este, el crecimiento de los aviones requeridos. Ello es así, porque es este segmento de ingresos los que viajan de manera recurrente, ya sea para vacaciones en el interior de sus regiones, o bien, por motivos de negocios. La tabla 1 muestra el crecimiento esperado de la clase media en el mundo, medido como millones de hogares. 
Tabla 1

Proyección del crecimiento de la clase media en el mundo, 2017-2037

\section{Clase Media Global}

Hogares con ingresos anuales entre \$20,000 y \$150,000 (precios constantes de PPP para 2017)

\begin{tabular}{lccc}
\hline \multicolumn{1}{c}{ Region } & 2017 & 2027 & 2037 \\
Africa & 216. & 347. & 527. \\
Asia-Pacifico & 1406. & 2071. & 2811. \\
CIS & 113. & 160. & 189. \\
Europa & 454. & 476. & 479. \\
America Latina & 348. & 437. & 520. \\
Medio Oriente & 187. & 231. & 259. \\
Norte América & 260. & 264. & 264. \\
& & & 5049. \\
Total Clase Media & 2984. & 3986. & 9010. \\
Total de Población & 7550. & 8330. & $56 \%$ \\
\% Clase Media & $40 \%$ & $48 \%$ & \\
\hline
\end{tabular}

Fuente: Oxford Economics, Airbus

No solo se estima que el número de vuelos y el número de aviones necesarios para satisfacer la creciente demanda aumente; también existen otros factores que harán crecer al tráfico aéreo en el futuro, como el número de mega ciudades que habrá en el mundo. La existencia de una mega ciudad implica un número de viajeros internacionales y locales mucho mayor en relación a otras ciudades; los factores que lo explican son principalmente: el crecimiento de centros de negocios internacionales, polos de atracción turística, mayores flujos comerciales y financieros, etc. La tabla 1 muestra la proyección del número de mega ciudades en el mundo en los próximos 20 años; definidas como las ciudades con más de 10,000 pasajeros diarios que arriban la ciudad por los distintos motivos señalados desde una distancia mayor de 20,000 millas ${ }^{1}$.

\footnotetext{
${ }^{1}$ Bajo esa definición, se espera que hasta el año 2032 México solo cuente con una mega ciudad, la Ciudad de México; para el año 2037, se espera que Cancún alcance esas dimensiones y se convierta en la segunda mega ciudad del país
} 
Tabla 2

Proyección del crecimiento de megas ciudades en el mundo

Número de mega ciudades en el mundo*

\begin{tabular}{ccccc}
\multicolumn{5}{c}{ AÑO } \\
\hline 2017 & 2022 & 2027 & 2032 & 2037 \\
64 & 70 & 76 & 84 & 91 \\
\hline
\end{tabular}

*Ciudades con más de 10,000 pasajeros diarios de larga distancia

(distancia de vuelo> 2,000 millas excluyendo el tráfico nacional)

Fuente : Global Market Forecast 2018, Airbus

Los datos anteriores son una muestra de las razones que fundamentan una perspectiva de crecimiento importante de la industria aeroespacial en el mundo durante los próximos años. El desarrollo de las estrategias de crecimiento por parte de las empresas del ramo a nivel mundial, en conjunción con el tipo específico de políticas comerciales y de desarrollo de capacidades endógenas e innovadoras formuladas a nivel país, determinará tanto los patrones de comercio aeroespacial a nivel global, como la distribución del valor que se genere en torno a la industria. En la siguiente sección, se describen los patrones de comercio aeroespacial a lo largo de las últimas dos décadas.

\section{Comercio mundial aeroespacial, 1995-2017}

La figura 5 muestra los patrones de comercio aeroespacial en 1995 utilizando la teoría de grafos. El grosor de los enlaces indica el tamaño del flujo comercial, medido en millones de dólares. La dirección del vector indica la dirección del flujo de exportaciones y quién recibe ese flujo en forma de importaciones. Para mostrar la importancia de cada país en el comercio mundial, el tamaño de la esfera muestra el saldo de balanza comercial en términos absolutos (exportaciones + importaciones) mientras que el color es un indicador del saldo; si es verde es superavitario, si es rojo, deficitario. 


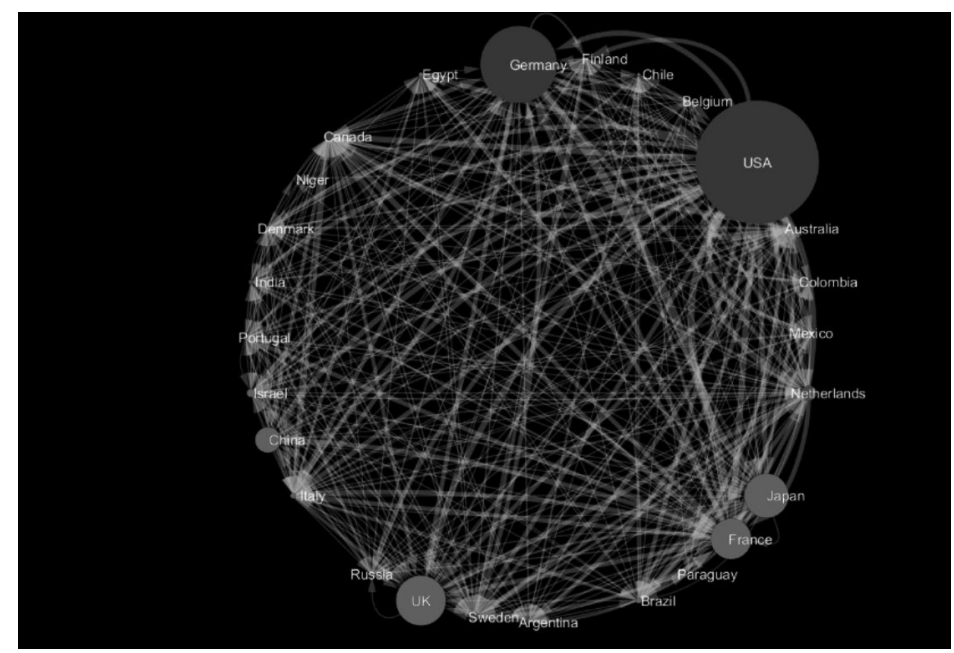

Figura 5. Balanza comercial y las transacciones económicas mundiales de la industria aeroespacial, 1995 Fuente: Elaboración propia con datos de UNCTAD STAT, 2018.

En la figura anterior se puede apreciar que Estados Unidos era el líder a nivel mundial, con un volumen de comercio 2.5 veces mayor que su más cercano competidor: Alemania. Uno de los patrones que sobresale es que ambos países son los exportadores a nivel mundial de valor agregado, o dicho en otros términos, la industria aeroespacial se concentraba en esos dos países.

Japón, Francia y Reino Unido, acompañan de manera lejana y deficitaria el comercio aeroespacial en el mundo, seguidos muy de lejos por China, con un volumen de comercio 15 veces menor con respecto al norteamericano. Por su parte, México para ese año no figura en la escena internacional; exportaba 316 millones de dólares, equivalentes al $0.5 \%$ del volumen de comercio aeroespacial mundial, con una participación equivalente al $0.5 \%$ del comercio mundial en el ramo. El 99.1\% de sus exportaciones para ese año, tenía destino Estados Unidos. La figura 6 muestra una radiografía del comercio aeroespacial en 2005. Como se puede apreciar, existen cambios de la más alta relevancia con respecto a los patrones comerciales existentes en 1995. Primero, Alemania crece prácticamente al mismo volumen de comercio de Estados Unidos, con la diferencia de que el primero continúa con un patrón superavitario, mientras que EEUU se convierte de un país superavitario en uno deficitario. Canadá se vuelve un actor relevante, ocupando el tercer lugar en volumen de comercio, también con superávit. Por su parte, Reino Unido y Francia pierden participación en el comercio mundial y China comienza a ser un actor relevante, superando a Francia, aunque con déficit. A nivel 
latinoamericano, Brasil, mediante una estrategia de creación de empresas locales apoyadas con recursos públicos, es por mucho, el actor más relevante de la región.

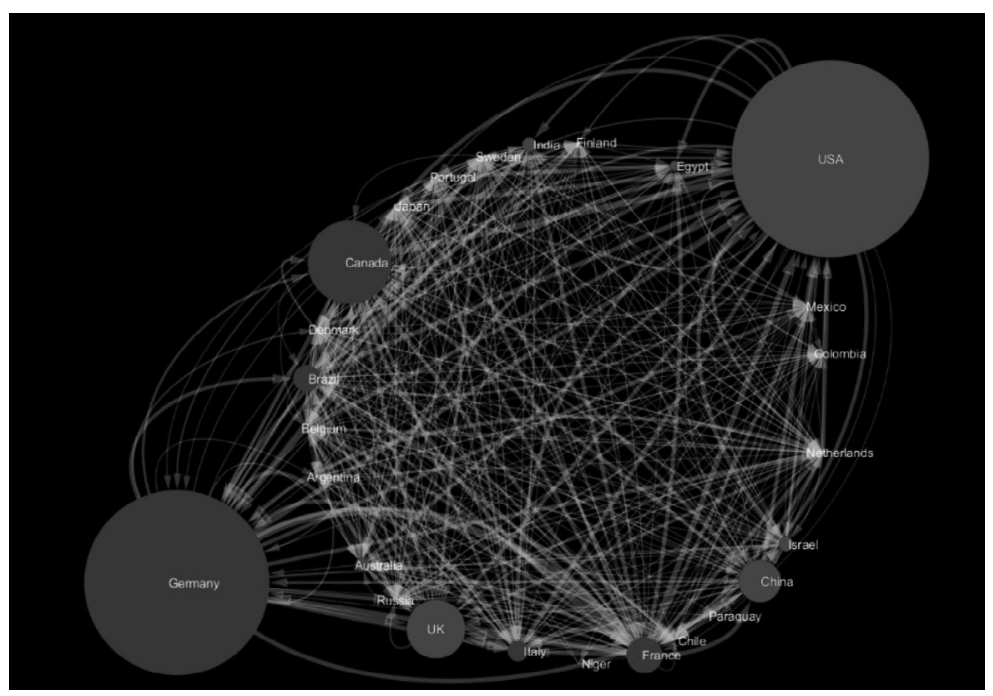

Figura 6. Balanza comercial y las transacciones económicas mundiales de la industria aeroespacial, 2005. Fuente: Elaboración propia con datos de UNCTAD STAT, 2018.

México diversifica sus destinos de exportación, ampliado sus redes comerciales en la industria de 11 países en 1995 a 17 en 2005, lo que genera ventas relativas marginalmente menores hacia EEUU, país hacia el cual exportaba en ese año el 95.8\% del total de sus exportaciones aeroespaciales. Sin embargo, su volumen de ventas al extranjero cae de 316, a 290 millones de dólares, una caída del volumen de comercio del ramo de $8 \%$ con respecto a 1995. Si se considera que el comercio mundial creció 20\%, la participación de México en el comercio mundial cae de $0.5 \%$ a $0.39 \%$.

Para el año de 2017, el comercio aeroespacial en el mundo crece $128 \%$ en relación a 2005, hecho que genera una recomposición significativa del escenario de comercio internacional de la IA. Estados Unidos vuelve a establecerse como la superpotencia comercial del mundo, si bien, resultado de establecer empresas en otros países aprovechando las ventajas de costos generadas a partir de la localización geográfica en otras latitudes que lo hace deficitario. Alemania continúa siendo el segundo lugar de volumen de comercio, con un constante superávit, lo que es reflejo de una estrategia productiva distinta, en donde las fases de alta generación de valor (investigación, diseño, y componentes de sistema) son producidas de manera interna. 


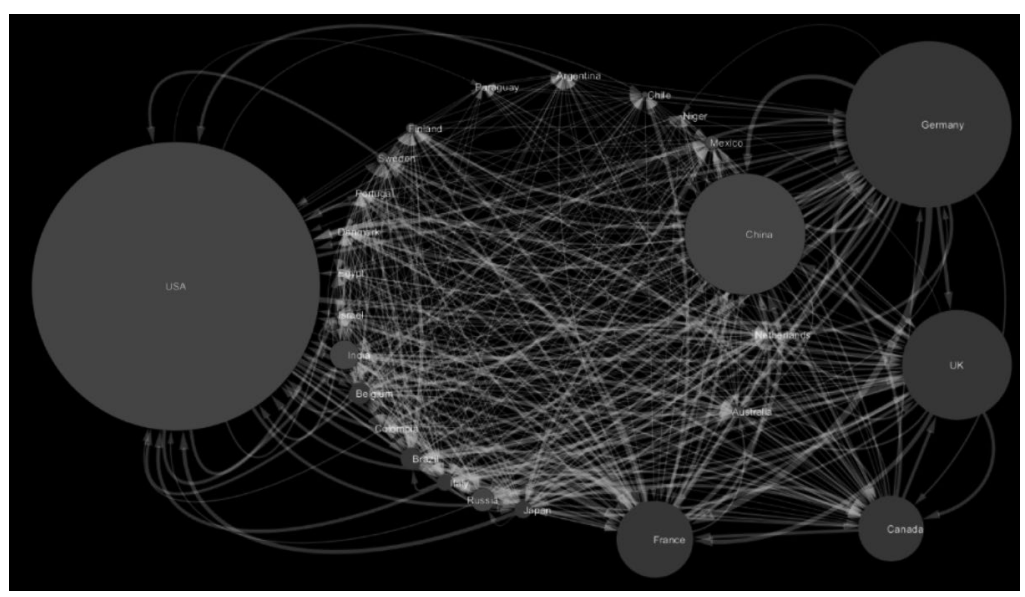

Figura 7. Balanza comercial y las transacciones económicas mundiales de la industria aeroespacial, 2017. Fuente: Elaboración propia con datos de UNCTAD STAT, 2018.

El crecimiento económico de China se ve reflejado en su volumen de comercio aeroespacial; en 2017 se convierte en la tercera potencia comercial de la industria, si bien, con importaciones mayores a su volumen de ventas al extranjero. Ello es resultado del seguimiento por parte del gigante asiático de una política industrial, acompañada de financiamiento (que generalmente proviene de sus propios bancos estatales) y de desarrollo de capacidades endógenas que le permitieron construir empresas capaces no solo de integrarse en una cadena, sino de competir dentro de ella con las empresas líderes de cada segmento. Reino Unido, Francia y Canadá, respectivamente, son los países que se ubican después de los mencionados anteriormente como las grandes potencias productoras y comerciales de bienes y servicios aeroespaciales.

Por su parte, el volumen comerciado por México también crece de manera importante (157\% en relación a 2005), crecimiento superior al mundial, lo que permite un crecimiento de la participación del comercio de México en el mundo. Además, se diversifican los destinos de comercio, pasando de tener 17 socios comerciales en 2005 a 23 en 2017. Sin embargo, a pesar de ubicarse como el décimo segundo productor aeroespacial a nivel mundial, la participación de México en la CGV y en el comercio aeroespacial del mundo sigue siendo, de la misma manera que a lo largo de todo el periodo analizado, marginal; la participación de México en el comercio mundial de la IA, fue de $0.5 \%$ en 1995 , de $0.39 \%$ en 2005 y de $0.44 \%$ en 2017.

Por su parte, la escasa participación de México en el contexto de la IA global, responde a la estrategia de integración a la CGV, ubicándose de inicio en la producción se segmentos de ensamblaje que representan la menor generación de valor y tratando de escalar en la cadena de producción. Una muestra clara de la imposibilidad de escalar en la cadena de valor con 
una estrategia de upgrading tecnológico como la seguida por México, se constituye en el hecho de que, a pesar de los esfuerzos específicos por elaborar componentes aeronáuticos de alto contenido mexicano, la generación de alto valor se encuentra protegida por derechos de propiedad e incluso, por acceso a conocimiento de procesos específicos en cada país. Considérese el caso de la empresa Bombardier Aeroespace en Querétaro con la construcción del Learjet 85; la planta ubicada en México se encarga de manufacturar el fuselaje, ensamblar las alas, los estabilizadores horizontales y verticales, así como fabricar e instalar los arneses eléctricos (ProMéxico, 2014); sin embargo, la investigación y el diseño se realizan fuera de México (principalmente en Canadá y Europa) y el ensamblaje final en Wichita, Estados Unidos.

Más allá de las posiciones específicas que ocupa cada uno de los países, interesa rescatar cuales han sido las políticas comerciales y de fomento industrial que han generados los cambios analizados hasta aquí, en particular en aquellos países en donde los cambios han sido más significativos.

La estrategia seguida por las firmas europeas y apoyadas por la política comercial de sus gobiernos, apostó por la reubicación geográfica de sus empresas en aquellas zonas que ofrecieran ventajas en términos de costos. En este punto el gran despegue de las tecnologías de información, aportó la base tecnológica para la descentralización productiva. De esta forma, para abastecer al mercado norteamericano, algunas empresas europeas se establecieron en lugares que les permitieran obtener ventajas en términos de costos, tanto en la producción como en la distribución a los lugares de ensamblaje o de consumo final. Los menores costos laborales, los acuerdos comerciales y la cercanía geográfica de México con EEUU, lo posicionaron como un lugar de destino de inversiones de la IA, para abastecer al mercado norteamericano, si bien, en productos muy específicos y de relativamente bajo valor agregado.

China pasa de ser un país de baja producción y comercio aeroespacial en 1995, a ser una potencia en el año 2017, con una estrategia diametralmente distinta. China comenzó atrayendo inversiones aeroespaciales desde principios de los años noventa, ofreciendo incentivos fiscales, y mano de obra altamente especializada. La estrategia de China, a diferencia de países como México, no era insertarse en el largo plazo en una cadena global de valor liderada por países ya productores, sino el desarrollo de capacidades endógenas mediante el uso de políticas industriales focalizadas, acceso a financiamiento, en un inicio mayoritariamente público y después con alianzas público-privadas para realizar el proceso completo de la cadena, desde la investigación, el desarrollo, el diseño, la producción, el ensamblaje, y la comercialización de los bienes finales de la IA. Uno de los resultados tangibles, es que la industria china no solo diseña, desarrolla y produce aviones, sino también de manera reciente, construyeron el Formosat-5, el primer satélite desarrollado íntegramente por la República de China. 
Por otra parte, los productores norteamericanos y canadienses, buscando ventajas de costos, han apostados porque sus OEMs, ya no integran todas las actividades de la cadena de valor del sector alrededor de sedes dentro de su territorio, sino que las confían a los suministradores de primer nivel, Tier I, mediante contratos con especificaciones técnicas muy ajustadas en otros territorios. (Oficina Económica y Comercial de la Embajada de España en Ottawa, 2018).

\section{Conclusiones}

El presente estudios tuvo por objetivo de analizar los cambios acaecidos en la IA mundial durante los últimos 24 años, así como las políticas nacionales que los generaron, mediante un abordaje teórico de CGV y el uso de teoría de grafos. Estos cambios son originados por modificaciones en las relaciones de comercio asociados a las ventajas comparativas y competitivas de cada país y fueron sustituidas por relaciones inter e intrafirma con un alto nivel de interdependencia, en la que el comercio de bienes intermedios y el intercambio global se organiza en forma de cadenas de valor. La gran explosión de las tecnologías de la información y comunicación aportó la base tecnológica para fomentar dichos cambios.

En esa dirección, la industria aeroespacial se integra en forma de cadenas globales de producción, con países que constituyen el centro productivo y dentro de los cuales se desarrollan las etapas de mayor generación de valor de la cadena, mismas que se encuentran protegidas por regulaciones en materia de derechos de propiedad, certificaciones y estandarización de procesos, y países satélites que cumplen en mayor o menor medida, funciones de Tier 1 o Tier 2 (tal es el caso de México). En este contexto, los países en desarrollo buscan integrarse a esas actividades productivas generando crecimiento interno a partir de la integración a las cadenas de valor, bajo el supuesto de que dicha integración produce derramas positivas mediante el aprendizaje y la creación de capacidades endógenas que les permitirán de forma gradual, escalar en la cadena de valor hacia actividades más rentables.

El estudio acopia evidencia relacionada con el hecho de que esta estrategia se encuentra acotada desde el origen y que las acciones de política pública basadas en el modelo upgrading profundizan las asimetrías y amplían la subordinación de las empresas y países no líderes, vía requerimientos estandarizados de procesos; a nivel de país, se produce cambios relativos en el liderazgo de los principales actores, pero no permite la entrada de nuevos competidores.

Un caso distinto es el de China, que pasa de ser un país de baja producción y comercio aeroespacial en 1995, a ser una potencia en el año 2017, siguiendo un modelo de crecimiento de la IA diametralmente distinto de la integración en CGV de forma tradicional. La forma de integración del país asiático fue mediante el desarrollo de capacidades endógenas no 
alineadas a los requerimientos de las firmas transnacionales, el uso de políticas industriales focalizadas, acceso a financiamiento proveniente principalmente de bancos Estatales para realizar el proceso completo de la cadena, desde la investigación, el desarrollo, el diseño, la producción, el ensamblaje, y la comercialización de los bienes finales de la IA.

El estudio muestra evidencia de los países que siguieron una estrategia de integración a las CGV buscando crecimiento en la cadena con una estrategia de upgrading, se han mantenido con participaciones marginales tanto en la producción como en el volumen de comercio mundial. Tal es el caso de México, cuya participación en el comercio aeroespacial mundial, fue de $0.5 \%$ en 1995 , de $0.39 \%$ en 2005 y de $0.44 \%$ en 2017.

Lo anterior muestra apenas indicios de que la estrategia para escalar en la cadena hacia actividades más rentables y de mayor generación de valor, debiera ser distinta a la del upgrading tradicional. La estrategia seguida por México para integrarse a la CGV de la IA, aunado a la falta de una política industrial y de desarrollo integral de capacidades endógenas. ha mantenido al país en los segmentos de bajo valor de la cadena, hecho que se ve reflejado en la prácticamente nula participación de México en el comercio internacional.

Visto desde una perspectiva más amplia, la estrategia competitiva de México se basa en salarios bajos para el sector (las remuneraciones medias de la IA rondan los 8 mil pesos mensuales), se complementa con una inexistente política industrial para el sector y con una baja inversión para el desarrollo de capacidades endógenas que permitan entender el ciclo completo de producción de las aeronaves; los sistemas institucionales de enseñanza se encuentran diseñados para atender requerimientos técnicos específicos de producción de empresas transnacionales, manteniéndose en la mayoría de los casos en niveles bajos de especialización; el 47\% de los programas de enseñanza corresponden a niveles de Técnico Bachiller, Técnico Básico y Técnico Superior Universitario, mientras que tan solo el 1\% corresponde a algún programa de maestría (ProMéxico, 2014), lo que muestra que la especialización no se genera en los segmentos altos de creación de conocimiento, lo que dificulta la innovación.

Si bien, México y China tienen amplias diferencias demográficas, económicas, territoriales y de acceso a recursos, algunas lecciones generales de la política industrial de China podrían ser aprendidas por México. En principio, el propio diseño de una política industrial que privilegie sectores claves para la economía no puede ser soslayado por México, como lo ha sido en los últimos 30 años. Si bien, es complicado que el financiamiento a esa política provenga del sector bancario (como ocurre en el caso de China con los bancos Estatales), es necesario destinar una parte de los recursos públicos al desarrollo de los sectores tecnológicamente avanzados; ello sería impensable si no se cuenta con mayores recursos fiscales que permitan el financiamiento de este tipo de políticas. 
De manera adicional, es necesario el desarrollo de una rama educativa de atención al sector aeroespacial no solo para atender las necesidades locales y de corto plazo de las empresas multinacionales ubicadas en México, sino que de manera gradual pero sostenida, cubra mayores y más elaboradas etapas del proceso de producción de la IA, como ocurre en el caso chino.

Ello debiera ser acompañado de una política de competencia no basada en bajos costos salariales, sino en dirección opuesta, que haga más atractiva la incorporación de mano de obra calificada al sector aeroespacial, que acompañe de cerca a la relativamente alta productividad del sector.

\section{Referencias}

AIA. (2017). 2017 Facts \& Figures. U.S. Aerospace \&Defense. Arlington: Aerospace Industries Association. Disponible en: https://www.aia-aerospace.org/report/2017-facts-figures/. Consultado: 3/abril/2019

AirBus (2018). Global market Forecast: Global Networks, Global Citizens., France, AirBus.

Brown-Grossman, F., y Dominguez, L. D. (2013). Can Mexico Set Up in the Aerospace and the Software and IT Global IT Value Chain as a High-Value-Added Player? Inter-American Development Bank. Integration and Trade Sector. Disponible en: https://publications.iadb.org/handle/11319/4265. Consultado: 27/abril/2019.

Casalet, M. (Ed.)(2013). La Industria Aeroespacial. Complejidad Productiva e institucional. México, FLACSO. ISBN9786079275112

Clearwater. (2011). Aerospace Global Report 2011. IMAP. Disponible en: http://d332c5czpwjztv.cloudfront.net/ wp-content/uploads/2011/02/Aerospace_Global_Report_2011_A41.pdf. Consultado: 25/junio/2018.

Cypher, J. M., y Pérez-Escalante, A. A. (2013). Instituciones y tecnologías como factores clave en los proyectos nacionales del desarrollo: una análisis comparativo de Brasil y México. Apuntes del CENES, Volúmen 32, No. 56, Julio-Diciembre, 105-138. Disponible en: https://www.redalyc.org/articulo.oa?id=479549579006. Consultado: 8/mayo/2019. https://dx.doi.org/10.19053/issn.0120-3053

Deloitte. (2018). On a asolid profitble growth path. 2018 Global aerospace and defense industry outlook. Deloitte. Disponible en: https://www2.deloitte.com/content/dam/Deloitte/us/Documents/manufacturing/us-mfg-2018global-a-and-d-sector-outlook.pdf. Consultado:26/mayo/2019.

Dicken, P. (2011). Global Shift: Mapping the Contours of the World Economy (Sexta ed.). London: Sage Publishing. http://dx.doi.org/10.25336/csp29369

Domínguez V.L., Brown-Grossman F., y Hernández C.J, (2018), Fuerzas motoras y obstáculos de la cadena aeroespacial en México. Facultad de Economía, UNAM.

Flores, S., y Villarreal, A. (2017). "Comparative Analysis of the Developmental Strategy of Aerospace Industry in Brazil, Canada and México: Public-Policy Implications”. Latin American Policy, 8(1), 41-62. https://doi. org/10.1111/lamp.12113

Gereffi, G. (1994). "The organization of buyer-driven global commodity chain: How U.S. retailers shape overseas production networks." En G. Gary, \& M. Korzeniewics, Commodity chains and global capitalism. London: PRAEGER. Disponible en: https://www.researchgate.net/profile/Gary_Gereffi/publication/281870191_The_ Organization_of_Buyer-Driven_Global_Commodity_Chains_How_US_Retailers_Shape_Overseas_Production_Networks/links/55fc801e08aec948c4b6b47a.pdf. Consultado: 28/marzo/2019.

Gereffi, G. (1999). "International trade and industrial upgrading in the apparel commodity chain." Journal of International Economics, 48(1), 37-70. https://doi.org/10.1016/s0022-1996(98)00075-0 
Gereffi, G. (2001). "Las cadenas productivas como marco analitico para la globalización." Problemas del Desarrollo, 32(125), 9-37. https://doi.org/10.22201/iiec.20078951e.2001.125.7389

Gereffi, G. (2005). “The global economy: organization, governance and development." En N. J. (eds), The handbook of economic sociology (págs. 160-182). Nueva York: Princeton University. https://doi. org/10.1515/9781400835584.160

Gereffi, G. (2018). "Políticas de desarrollo productivo y escalamiento: la necesidad de vincular empresas, agrupamientos y cadenas de valor." En E. (. Peters, Cadenas Globales de Valor. Metodología, Teoría y Debates. (págs. 13-44). México: Universidad Nacional Autónoma de México. Facultad de Economía.

Gereffi, G., y Hernández-Stark, K. (2016). Global Value Chain: A primer (Segunda ed.). Carolina del Norte, Estados Unidos: Center on Globalization, Governance and Competitiveness, Social Science Research Institute. Disponible en: https://gvcc.duke.edu/cggclisting/global-value-chain-analysis-a-primer-2nd-edition/. Consultado: 10/abril/2019.

Hualde, A., y Carrillo, J. (2007). La industria aeroespacial en Baja California: características productivas y competencias laborales y profesionales. México: Colegio de la Frontera Norte.

KPMG International. (2016). Global Aerospace and Defense Outlook. Amstelveen: KPMG International.

López Galindo, M., y Pérez Rueda, S. O. (2018). Surgimiento y Crecimiento de la Industria Aeroespacial en México. Reporte de Investigación del Proyecto ¿Existe la Responsabilidad Social en las MiPymes de la industria Aeroespacial del Estado de Querétaro, México? México: Universidad Autónoma Metropolitana. Unidad Azcapotzalco. Disponible en: https://repository.uaeh.edu.mx/revistas/index.php/tepexi/article/view/2970/3035. Consultado: 10/abril/2019.

Mitchell, M. (2009). Complexity: A Guided Tour. Oxford University Press.

Newman, M. (2018). Networks. Oxford University Press.

Niosi, J., \& Zhegu, M. (2005). “Aerospace Clusters: Local or Global Knowledge Spillovers.” Industry and Innovation, 12(1), 1-25. https://doi.org/10.1080/1366271042000339049

Ortiz-Ospina, E., y Roser, M. (2018). International Trade Our World in Data. Disponible en: https://ourworldindata.org/international-trade. Consultado: 26/mayo/2019.

ProMéxico (2014), Plan Nacional de Vuelo, Mapa de ruta de la industria aeroespacial mexicana, CDMX, ProMéxico. Disponible en: https://www.gob.mx/cms/uploads/attachment/file/60149/MRT-Aeroespacial-2014.pdf. Consultado: 7/abril/2019.

Salinas-García, R. J. (2012). "Desarrollo Industrial y Formación Profesional en la industria aeronáutica en Querétaro." Revista Educación y Desarrollo, 5-14.

Sandoval, S. (2013). La cadena global de hortalizas. La estrategia de ascenso de los productores sinaloenses. México: Instituto de Investigaciones Económicas. UNAM.

Secretaria de Economía. (2017). Pro Áreo 2.0. Programa Estratégico de la Industria Aeroespacial. México: Secretaria de Economía. Gobierno Federal. Disponible en: http://economia.gob.mx/files/comunidad_negocios/ industria_comercio/PROAEREO-12-03-2012.pdf. Consultado: 13/mayo/2019.

Sturgeon, T. (2011). "De cadenas de mercancías (commodities) a cadenas de valor". Eutopía, Revista De Desarrollo Económico Territorial, n. ${ }^{\circ} 2$ (noviembre), 11-38. https://doi.org/https://doi.org/10.17141/eutopia.2.2010.1027.

Sturgeon, T., \& Memedovic, C. (2011). Mapping Global Value Chains: Intermediate goods trade and structural change in hte world economy. Working Paper. Disponible en: https://open.unido.org/api/documents/4811381/ download/Mapping\%20Global\%20Value\%20Chains\%20-\%20Intermediate\%20Goods\%20Trade\%20 and $\% 20$ Structural $\% 20$ Change $\% 20$ in $\% 20$ the $\% 20$ World\%20Economy. Consultado: 17/abril/2019.

Sturgeon, T., Gereffi, G., Guinn, A., \& Zylberberg, E. (2013). Brazilian Manufacturing in International Perspective. A Global Value Chain Analysis of Brazil's Aerospace, Medical Devices, and Electronics Industries. Sao Paulo: Prepared for Brazil's Confederação Nacional da Indústria (CNI). Disponible en: https://gvcc.duke.edu/cggclisting/brazilian-manufacturing-in-international-perspective-a-global-value-chain-analysis-of-brazils-aerospace-medical-devices-and-electronics-sectors/ 


\section{H. E. Díaz Rodríguez, et al. / Contaduría y Administración 65(4), 2020, 1-24}

http://dx.doi.org/10.22201/fca.24488410e.2020.2418

Vázquez M.A y Bocanegra C. "La industria aeroespacial en México: características y retos en Sonora" en Revista Problemas del Desarrollo, Volumen 49, Número 195, octubre-diciembre 2018. Pp. 153-176. https://doi. org/10.22201/iiec.20078951e.2020.202

Wasserman, S., \& Faust, K. (1994). Social Network Analysis: Methods and Applications (Vol. 8). Cambridge University Press

Williamson, O. E. (1975). Markets and Hierarchies: Analysis and Atiturust Implications. Nueva York: Free Press. https://doi.org/10.2307/2230812

Williamson, O. E. (1979). "Transaction-Cost Economics: The Governance of Contractual Relations." Journal of Law and Economics, 22(2), 233-261. https://doi.org/10.1086/466942.

Williamson, O. E. (1985). The Economics Institutions of Capitalism. Nueva York: Free Press. 